\title{
Tolerância ao calor em bovinos das raças Nelore branco, Nelore vermelho e Pantaneira
}

\author{
Heat tolerance in Nelore branco, Nelore vermelho and Pantaneira breeds in the \\ Pantanal region, Brazil
}

\author{
BARBOSA, Bruna Rocha Passos ${ }^{1 *}$; SANTOS, Sandra Aparecida ${ }^{2}$; ABREU, Urbano \\ Gomes Pinto de ${ }^{2}$; EGITO, Andrea Alves ${ }^{3}$; COMASTRI FILHO, José Aníbal²; \\ JULIANO, Raquel Soares ${ }^{2}$; PAIVA, Samuel Rezende ${ }^{4}$; McMANUS, Concepta ${ }^{5}$
}

\author{
${ }^{1}$ Universidade de São Paulo, Faculdade de Medicina Veterinária e Zootecnia da São Paulo, Departamento \\ de Medicina Veterinária Preventiva e Saúde Animal, São Paulo, Brasil. \\ ${ }^{2}$ Embrapa Pantanal, Corumbá, Mato Grosso do Sul, Brasil. \\ ${ }^{3}$ Embrapa Gado de Corte, Campo Grande, Mato Grosso do Sul, Brasil. \\ ${ }^{4}$ Embrapa, Secretaria de Relações Internacionais, Brasília, Distrito Federal, Brasil. \\ ${ }^{5}$ Universidade de Brasília, Faculdade de Agronomia e Veterinária, Brasília, Distrito Federal, Brasil. \\ *Endereço para correspondência: bruna888@ hotmail.com
}

\section{RESUMO}

A identificação de parâmetros/ métricas associados à adaptação ambiental é uma das principais tendências e desafios da pecuária mundial. Desta forma, o objetivo deste trabalho foi avaliar as características de tolerância ao calor de bovinos da raça Nelore e Pantaneiro no ecossistema do Pantanal matogrossense no pico de calor da região. Estes animais foram mantidos por duas horas à sombra $(8: 00 \mathrm{~h}$ às 10:00h) e em seguida uma hora ao sol (10:00h às 11:00h). Após a exposição foram analisados parâmetros de frequência cardíaca (FC), frequência respiratória (FR), temperatura retal (TR), temperatura superficial da costela (TSC), temperatura superficial do pescoço (TSP) e temperatura superficial da garupa (TSG). Também foram avaliados a taxa de sudação (TXS), comprimento e densidade de pelos e calculados o índice de tolerância ao calor (ITC). No período estudado, a temperatura ambiental variou de 29,0 a $40,0^{\circ} \mathrm{C}$ e a média para umidade relativa foi de $75 \%$. O ambiente teve influência marcante sobre as variáveis fisiológicas, com exceção da FR. A TR e a TXS diferiram significativamente entre os grupos genéticos, sendo da raça Nelore mais elevada em relação à Pantaneira, mas todas dentro dos limites fisiológicos. A análise multivariada mostrou a importância de variáveis como taxa de sudação e comprimento de pelos para adaptação de bovinos na região. Apesar do Nelore vermelho estar separado dos outros dois grupos, foi possível concluir que ambas as raças analisadas apresentaram capacidade de adaptação e tolerância ao calor às condições climáticas do Pantanal e podem ser produzidas de modo sustentável na região.

Palavras-chave: Bos indicus, Bos Taurus, estresse calórico, frequência cardíaca, temperatura retal

\section{SUMMARY}

Identification of parameters/metrics associated with environmental adaptation is one of the main trends and challenges for livestock worldwide. Thus, this study was conducted at Nhumirim Farm, in Nhecolândia, Pantanal, during the month of February, period with highest ambient temperatures. We used five cattle of each genetic group (White Nelore, Red Nelore and Pantaneira) to evaluate characteristics related to heat tolerance. Heart rate (HR), respiratory rate (RR), rectal temperature (RT), skin temperature (ST), sweating rate (SR), morphometric measurements, hair length (HL) and heat tolerance index (HTI) were evaluated. During the study the air temperature ranged from 29.0 to $40.0^{\circ} \mathrm{C}$ and the average relative humidity was $75 \%$. RT and ST were highest in White Nelore, followed by Red Nelore and 
Pantaneira. The average HTI value for the three groups was between 9 and 10, indicating high tolerance. The environment had considerable effect on physiological variables, except RR, RT and SR that significantly differed between genetic groups, with Nelore being higher than the Pantaneira, but all within physiological limits. Multivariate analysis showed the importance of variables such as sweating rate and hair length for the adaptation of cattle in the region. The red Nelore separated from the other two groups. We conclude, therefore, that both zebu (white and red Nelore) and European (Pantaneira) showed adaptability and heat tolerance to climatic conditions of the Pantanal.

Keywords: Bos indicus, Bos taurus, heat stress, heart rate, rectal temperature

\section{INTRODUÇÃO}

A produtividade de bovinos de corte em pastagem nas regiões tropicais está diretamente relacionada à capacidade de adaptação às condições ambientais, sendo a tolerância ao calor um dos aspectos mais importantes neste processo (MAGALHÃES et al., 2000; McMANUS et al., 2009). A produtividade máxima só pode ser atingida se os animais forem mantidos na zona de termoneutralidade (ZTN), a qual consiste em uma faixa de temperatura que confere conforto térmico em que há um gasto mínimo de energia para manter a homeotermia (SILVA, 2000). Esta faixa depende de alguns fatores, dentre os quais a capacidade de adaptação dos animais ao ambiente. Os zebuínos são mais tolerantes ao calor em relação aos taurinos devido, principalmente, ao processo evolutivo destas raças, que proporcionou o aparecimento de alelos relacionados à termotolerância (HANSEN, 2004), com habilidade termorregulatória superior em decorrência da taxa metabólica mais baixa, bem como da maior capacidade de perda de calor para o ambiente. No entanto, raças taurinas também podem desenvolver capacidade adaptativa às condições tropicais, como é o caso de diversas raças naturalizadas brasileiras, introduzidas no país por colonizadores ibéricos, e que se adaptaram ao ambiente por meio da seleção natural durante séculos (McMANUS et al., 2009).

Na região do Pantanal, predomina a criação extensiva de gado de corte, sendo a raça Nelore (Bos taurus indicus), a dominante. Por outro lado, a raça Pantaneira (Bos taurus ibericus), que foi a base da economia da região no século passado, está restrita a poucos núcleos de criação (JULIANO et al., 2011). O interesse em conhecer as respostas adaptativas desses animais às condições do Pantanal fundamenta-se na realidade da pecuária pantaneira, inclusive relatada por Egito et al. (2002), em que se observa uma forte tendência à substituição dos tipos locais de alta capacidade de adaptação ao ambiente, por raças mais produtivas, geralmente desenvolvidas em regiões de clima temperado, colocando as raças nativas sob risco de extinção. Há também o fato de que as raças de bovinos naturalizados podem constituir um material genético valioso para utilização em sistemas de produção alternativos ou programas de melhoramento para condições ambientais extremas (MAZZA et al., 1989).

Objetivou-se com este trabalho avaliar as características de tolerância ao calor de bovinos Bos indicus (Nelore branco e Nelore vermelho) e Bos taurus (Pantaneira) nas condições ambientais do Pantanal. 


\section{MATERIAL E MÉTODOS}

O trabalho foi desenvolvido na Fazenda Nhumirim, pertencente à Embrapa Pantanal, no município de Corumbá, Mato Grosso do Sul, a $18^{\circ} 59^{\prime} \mathrm{S}$ e $56^{\circ} 39^{\prime} \mathrm{W}$. A fazenda possui área de 4390,6 ha e está localizada na subregião da Nhecolândia, que corresponde a $19,48 \%$ da área total do Pantanal. O clima da região é do tipo AW, segundo a classificação de Köppen. De acordo com Soriano \& Galdino (2002), no Pantanal da Nhecolândia, a precipitação pluvial média anual fica em torno de $1.200 \mathrm{~mm}$. A temperatura média anual é de $25,5^{\circ} \mathrm{C}$, variando de 20,8 a $28,0^{\circ} \mathrm{C}$, mas quando se considera a temperatura média mensal das máximas, esta varia de 28,3 a $34,0^{\circ} \mathrm{C}$, sendo que nos meses de setembro a fevereiro as máximas absolutas ultrapassam $40,0^{\circ} \mathrm{C}$. A umidade relativa média anual é de $82 \%$, oscilando entre 75 e $86 \%$. (SANTOS et al., 2005).

O estudo foi realizado na estação quente (fevereiro), utilizando cinco animais de cada um dos seguintes grupos genéticos: Pantaneiro, Nelore branco e Nelore vermelho, totalizando 15 animais, sendo todas fêmeas não lactantes, entre cinco a dez anos de idade e criados em sistema extensivo com pastagem nativa e suplementação mineral (sal comum e mistura mineral para bovinos). $\mathrm{O}$ peso médio dos bovinos foi de 376,8; 396,2 e $282 \mathrm{~kg}$ para Nelore branco, Nelore vermelho e Pantaneiro, respectivamente. $\mathrm{O}$ número de repetições foi calculado de acordo com Kaps \& Lamberson (2009), que consideram o número mínimo necessário com um nível de significância de $95 \%$ e um poder do teste de $80 \%$. A determinação do índice de tolerância ao calor (ITC) baseou-se no método proposto por Baccari Junior (1986).
Para a avaliação das medidas fisiológicas, corporais e da pele (superficial), os animais foram conduzidos ao curral onde foram mantidos durante duas horas à sombra (10:00 às 12:00h), e em seguida por uma hora sob radiação solar direta (12:00 às 13:00h), tendo seus parâmetros fisiológicos aferidos após cada etapa. Foram mensurados os seguintes parâmetros: frequência cardíaca (FC), frequência respiratória (FR), temperatura retal (TR), temperatura superficial da costela (TSC), temperatura superficial da tábua do pescoço (TSP) e temperatura superficial da garupa (TSG). Com o valor das médias obtidas para as temperaturas retais (sombra $=\mathrm{TR} 1 \mathrm{e}$ sol = TR2), obteve-se o índice de tolerância ao calor por meio da fórmula: ITC $=10$ - (TR2 - TR1), em que, em uma escala de zero a dez, quanto mais próximo de dez for o índice, mais tolerante é o animal. Estas medidas foram repetidas após uma semana, gerando duas repetições para cada animal. No momento da realização do teste, os animais permaneceram em jejum e sem acesso à água.

A FC foi aferida com auxílio de um estetoscópio e a FR de acordo com o número de movimentos respiratórios da caixa torácica, conforme descrito por Feitosa et al. (2004). A TR foi determinada através de termômetro clínico e a TS com um termômetro infravermelho a laser que era acionado em quatro locais no animal: costela flanco esquerdo (TSC), tábua do pescoço esquerdo (TSP), garupa (TSG) e região axilar no membro torácico direito (TSA) a uma distância aproximada de dois metros. Determinouse a temperatura ambiente com auxilio de um termômetro de máxima e mínima e os valores de umidade relativa foram estimados a partir dos dados obtidos na estação meteorológica da Fazenda 
Nhumirim, de acordo com o horário das avaliações.

A taxa de sudação (TXS) foi avaliada conforme método desenvolvido por Berman (1957) e modificado por Schleger \& Turner (1965), que se baseia na mudança da coloração de discos de papel de filtro embebidos em cloreto de cobalto. Para o teste, foram utilizados discos de papel de filtro, assim obtidos por meio de uma punção vazadora do tipo usada para furar papel ou couro embebido em solução de cloreto de cobalto a $10 \%$. Três desses discos foram montados sobre lâmina de microscópio (três em cada lâmina) e nela fixados com fita adesiva transparente, tipo "durex"; a lâmina foi guardada em frasco hermeticamente fechado, contendo secante (sílica-gel) para proteção contra a umidade do ar. Os discos foram preparados 24 horas antes de serem usados, conforme recomendação de Silva et al. (2000). O local escolhido para o teste foi a região do flanco, cuja pele foi raspada com gilete para que a fita adesiva com os três discos fosse aplicada sobre esta região, iniciando-se imediatamente a cronometragem do tempo (em segundos) necessária à completa mudança da cor de cada disco, de azul-violeta para róseoclaro, sendo que cada disco era controlado em separado e calculada a média dos três (SILVA et al. 2000) Após cálculo do tempo (t), a taxa de sudação pode ser determinada de acordo com a fórmula: $\mathrm{S}\left(\mathrm{g} \cdot \mathrm{m}^{-2} \mathrm{~h}^{-1}\right)=38446,6 / \mathrm{t}$, conforme Silva et al. (2000). O teste da taxa de sudação foi realizado duas vezes para cada animal, e calculada a média dos valores obtidos.

$\mathrm{O}$ número e comprimento de pelos também foram determinados. As amostras destes foram coletadas na região esquerda da garupa (anca), com auxílio de uma pinça comum, de aproximadamente $5,0 \mathrm{~cm}$ de comprimento, e um molde feito com papel cartão, cuja área de
$0,5 \mathrm{~cm}^{2}$. Os valores foram convertidos para número de pelos $/ \mathrm{cm}^{2}$. Os pelos foram acondicionados em sacos de papel, devidamente identificados com o número e o grupo genético do animal, e depois espalhados sobre folha de papel branca, para serem contados, com auxílio de lupa e contador. Os dez maiores de cada amostra foram separados e medidos, conforme procedimento descrito por Bianchini et al. (2006).

A análise de variância foi feita para avaliar a influência dos efeitos fixos sobre as variáveis fisiológicas e parâmetros de pêlos usando o PROC GLM do programa SAS (SAS, 2010). No modelo foram incluídos os efeitos de grupo genético (Nelore branco, Nelore vermelho e Pantaneira), dia, animais e ambiente (sombra e sol) e a interação grupo genético $\mathrm{x}$ ambiente. Comparações entre médias foram feitas pelo teste de Tukey-Kramer a 5\%. Para as variáveis taxa de sudação, comprimento de pelos e tamanho de pelos foram incluídos somente grupo genético e animais e as comparações entre médias foi feita pelo teste de Tukey, baseado na amplitude total estudentizada. Outras análises incluíram análise de componentes principais, análise discriminante canônica (CANDISC), bem como distância euclidiana entre grupos genéticos (PROC CLUSTER; TREE). Medidas de desempenho não foram incluídas, pois o estudo foi executado somente com o objetivo de avaliar a tolerância ao calor e em um período curto de tempo.

\section{RESULTADOS E DISCUSSÃO}

No período estudado, a temperatura ambiental (TA) variou de 29,0 a $40,0^{\circ} \mathrm{C}$ e a umidade relativa (UR) de 65 a $80 \%$ para o mês de fevereiro. A zona de 
conforto para bovinos é relativamente pequena, sendo que para as raças européias está entre $-1^{\circ} \mathrm{C}$ e $16^{\circ} \mathrm{C}$ e para as raças zebuínas está entre $10^{\circ} \mathrm{C}$ e $27^{\circ} \mathrm{C}$ (SANTOS et al., 2005), ou seja, os elevados índices de TA na região do Pantanal representam um grande desafio aos animais, exigindo que desenvolvam mecanismos adaptativos para dissipação do calor. Dos fatores fixos analisados, o ambiente teve influência marcante sobre as variáveis fisiológicas, com exceção da FR (Tabela 1).

Tabela 1. Resumo da análise de variância das características fisiológicas nas raças naturalizadas do Pantanal

\begin{tabular}{lcccccccc}
\hline Item & FC & FR & TR & TSC & TSP & TSG & TSA & TXS \\
\hline CV & 1,4 & 14,3 & 0,69 & 2,60 & 1,82 & 2,87 & 3,42 & 25,32 \\
Média & 63,4 & 27,2 & 39,3 & 33,6 & 33,5 & 34,3 & 32,6 & 225,5 \\
Grupo genético & $\mathrm{ns}$ & $\mathrm{ns}$ & $* *$ & $\mathrm{~ns}$ & $\mathrm{~ns}$ & $\mathrm{~ns}$ & $\mathrm{~ns}$ & $* *$ \\
Dia & $\mathrm{ns}$ & $\mathrm{ns}$ & $\mathrm{ns}$ & $* *$ & $* *$ & $* *$ & $* *$ & $\mathrm{~ns}$ \\
Ambiente & $*$ & $\mathrm{~ns}$ & $* *$ & $* *$ & $* *$ & $* *$ & $* *$ & $* *$ \\
Animal & $* *$ & $* *$ & $* *$ & $\mathrm{~ns}$ & $*$ & $\mathrm{~ns}$ & $\mathrm{~ns}$ & $\mathrm{~ns}$ \\
Grupo genético x ambiente & $\mathrm{ns}$ & $\mathrm{ns}$ & $\mathrm{ns}$ & $\mathrm{ns}$ & $\mathrm{ns}$ & $\mathrm{ns}$ & $\mathrm{ns}$ & $\mathrm{ns}$ \\
\hline Pantaneira & $61,8^{\mathrm{a}}$ & $27,8^{\mathrm{a}}$ & $38,9^{\mathrm{b}}$ & $33,7^{\mathrm{a}}$ & $33,6^{\mathrm{a}}$ & $34,3^{\mathrm{a}}$ & $32,5^{\mathrm{a}}$ & $187,9^{\mathrm{a}}$ \\
Nelore branco & $61,2^{\mathrm{a}}$ & $25,7^{\mathrm{a}}$ & $39,5^{\mathrm{a}}$ & $33,3^{\mathrm{a}}$ & $33,5^{\mathrm{a}}$ & $34,3^{\mathrm{a}}$ & $32,6^{\mathrm{a}}$ & $259,8^{\mathrm{b}}$ \\
Nelore vermelho & $68,4^{\mathrm{a}}$ & $28,3^{\mathrm{a}}$ & $39,6^{\mathrm{a}}$ & $33,6^{\mathrm{a}}$ & $33,6^{\mathrm{a}}$ & $34,2^{\mathrm{a}}$ & $33,0^{\mathrm{a}}$ & $228,7^{\mathrm{b}}$ \\
\hline Sombra & $60,3^{\mathrm{b}}$ & $27,3^{\mathrm{a}}$ & $39.2^{\mathrm{b}}$ & $33,1^{\mathrm{b}}$ & $33,2^{\mathrm{b}}$ & $33,7^{\mathrm{b}}$ & $32,0^{\mathrm{b}}$ & - \\
Sol & $67,^{\mathrm{a}}$ & $27,2^{\mathrm{a}}$ & $39.5^{\mathrm{a}}$ & $34,0^{\mathrm{a}}$ & $33,7^{\mathrm{a}}$ & $34,9^{\mathrm{a}}$ & $33,4^{\mathrm{a}}$ & - \\
\hline Valores de referência & $50-80^{1}$ & $10-30^{2}$ & $37.9^{-3} 39.5^{3}$ & - & - & - & - & - \\
\hline
\end{tabular}

$\mathrm{FC}=$ frequência cardíaca $(\mathrm{bpm}) ; \mathrm{FR}=$ frequência respiratória $(\mathrm{mpm}) ; \mathrm{TR}=$ temperatura retal $\left({ }^{\circ} \mathrm{C}\right) ;$ $\mathrm{TSC}=$ temperatura superficial da costela $\left({ }^{\circ} \mathrm{C}\right) ; \mathrm{TSP}=$ temperatura superficial da tábua do pescoço $\left({ }^{\circ} \mathrm{C}\right)$; $\mathrm{TSG}=$ temperatura superficial da garupa $\left({ }^{\circ} \mathrm{C}\right) ; \mathrm{TSA}=$ temperatura superficial da axila $\left({ }^{\circ} \mathrm{C}\right) ; \mathrm{TXS}=$ taxa de sudação ((g.m-2h-1); ns = não significativo; * e **Significativo a 5 e $1 \%$ de probabilidade, respectivamente.

${ }^{1}$ Silva (2000); ${ }^{2}$ Kabuga (1992); ${ }^{3}$ Wenz et al. (2011).

Médias com letras diferentes, na coluna, diferem estatisticamente $(\mathrm{P}<0,05)$ pelo teste de Tukey-Kramer.

Em temperaturas altas, o principal mecanismo utilizado pelos animais para a perda de calor é a evaporação que ocorre na superfície da pele e pela via respiratória. $\mathrm{O}$ fato de a $\mathrm{FR}$ não ter sofrido influência do ambiente, em todas as raças avaliadas, sugere uma capacidade das mesmas em realizar a troca de calor via evaporação (suor), não necessitando alterar os demais parâmetros fisiológicos para manter a homeotermia.

A temperatura retal (TR) esteve dentro dos limites de normalidade descritos por Robinson (1999), variando de 38,9
(Pantaneira) a 39,6 graus (Nelore vermelho). Como a TR é frequentemente utilizada como índice de adaptabilidade em ambientes quentes, dependente do equilíbrio entre o ganho e a perda de calor, os valores obtidos neste estudo sugerem que os bovinos apresentaram adaptação às condições ambientais no período avaliado. De acordo com Furtado et al. (2012), a zona de conforto térmico para zebuínos está na faixa de 10 a $27{ }^{\circ} \mathrm{C}$ com limite crítico a partir de $35^{\circ} \mathrm{C}$, enquanto que para o gado europeu a zona de conforto varia de 0 a $16^{\circ} \mathrm{C}$ e se 
torna crítica a partir de $27^{\circ}$. Em temperaturas ambientes ao redor de $25^{\circ} \mathrm{C}$, ambas as raças apresentam temperatura retal ao redor de $38,5^{\circ} \mathrm{C}$ (VERCOE \& FRISCH, 1992). Por outro lado, McDowell et al. (1954) afirmam que bovinos de todas as raças apresentam temperatura retal média de $38,3^{\circ} \mathrm{C}$ com algumas variações, o que corrobora os achados do presente estudo. No entanto, a TR diferiu significativamente entre os grupos genéticos, sendo as do Nelore branco e vermelho relativamente mais elevada em relação ao Pantaneiro (Tabela 1). Estes resultados são divergentes dos obtidos para as mesmas raças nas condições ambientais de cerrado, na região de Brasília, Distrito Federal (McMANUS et al., 2009), bioma com clima tropical semelhante ao Pantanal, porém com variações na temperatura e umidade. Se a umidade do ar é baixa, a evaporação é facilitada, caso contrário, a evaporação será lenta ou mesmo nula (WILSON et al., 2007). Diante dessas respostas diferenciadas, Façanha et al. (2013) propuseram uma metodologia para avaliar a adaptação dos grupos genéticos às peculiaridades climáticas da região tropical, fundamentada em uma matriz multifatorial. Os valores de taxa de sudação (TXS) também foram influenciados pelo grupo genético (Tabela 1), sendo os valores da raça Nelore branco superiores aos do bovino Pantaneiro e até mesmo do Nelore vermelho, o que significa uma produção de suor mais rápida (em segundos) e, portanto, uma resposta térmica mais eficiente. Os valores médios da taxa de sudação $\left(\mathrm{g} . \mathrm{m}^{-2} \mathrm{~h}^{-1}\right)$ foram 259,$8 ; 228,7 \mathrm{e}$ 187,9 para Nelore branco, Nelore vermelho e Pantaneira, respectivamente. Em trabalhos realizados por Bianchini et al. (2006) e McManus et al. (2009) com bovinos na região de Brasília, a raça Pantaneira apresentou taxa de sudação maior que a Nelore, inclusive maior que os valores obtidos no presente estudo. Provavelmente, estas respostas diferenciadas devem-se a interação genótipo $\mathrm{x}$ ambiente, conhecida como plasticidade fenotípica (VIA \& LANDE, 1985), ainda pouco estudados na região.

A temperatura superficial (TS), avaliada em diferentes partes do corpo dos animais não diferiu entre os grupos genéticos, porém estas foram influenciadas pelo ambiente e dia de avaliação. Conforme esperado, a temperatura superficial dos animais se eleva ao sol devido ao decréscimo do gradiente térmico entre a superfície e o meio ambiente, diminuindo a perda de calor pelos mecanismos de radiação e convecção (SOUZA et al., 2006), consequentemente, dissipando calor principalmente por mecanismos evaporativos (respiração e sudação), que podem refletir nos parâmetros da frequencia respiratória e taxa de sudação.

Os valores médios de ITC para as raças zebuínas $(9,8)$ e Pantaneiras $(9,6)$ foram próximos de 10, indicando que apesar da raça Pantaneira apresentar valor pouco menor, ambas mostraram ser altamente tolerantes ao calor. Os resultados obtidos neste estudo são similares aos relatados por Titto et al. (2006) para raças zebuínas, de 9,70, como também aos obtidos por Souza et al. (2006) que observaram valor médio de 9,83 para a raça Sindi, também de origem indiana, no semiárido nordestino. Para as raças européias, Titto et al. (2006) encontraram valor de 9,52 para a raça Angus, pouco abaixo do observado para a raça Pantaneira. Porém, Vieira et al. (2003) encontraram valor de 9,70 para a raça Caracu em São Paulo.

A influência do grupo genético foi observada ainda nas características do 
pelame (Tabela 2). Segundo Bianchini et al. (2006), o pelame do animal é uma das características relacionadas à adaptação ao meio, em razão da função de fronteira entre o animal e o ambiente físico circundante, que interfere na resposta dos animais ao ambiente. Neste estudo, observamos que o número de pelos foi maior para o Nelore branco seguido do Pantaneiro e Nelore vermelho. O comprimento dos pelos foi maior para o Pantaneiro seguido do Nelore branco e do Nelore vermelho. O comprimento menor dos pelos representa uma vantagem em ambientes quentes, já que pelos longos, como os da raça Pantaneira, dificultam a transferência de calor a partir da capa, devido à barreira física oferecida pelas fibras.

Tabela 2. Resumo da análise de variância das características dos pelos, tempo e taxa de sudação nas raças naturalizadas do Pantanal

\begin{tabular}{lcc}
\hline Item & $\mathrm{NP}$ & $\mathrm{CP}$ \\
\hline $\mathrm{CV}$ & 17,70 & 32.35 \\
Média & 410,59 & 0,91 \\
Grupo Genético (GG) & $* *$ & $* *$ \\
Animal dentro de GG & $* *$ & $1.16^{\mathrm{a}}$ \\
\hline Pantaneira & $421,60^{\mathrm{a}}$ & $0.78^{\mathrm{b}}$ \\
Nelore branco & $462,44^{\mathrm{a}}$ & $0.77^{\mathrm{b}}$ \\
Nelore vermelho & $352,25^{\mathrm{b}}$ & e $1 \%$ de \\
\hline NP=número de pêlos/cm ${ }^{2} ; \mathrm{CP}=$ comprimento dos & pêlos $(\mathrm{mm}) ; *$ e $* *$ & Significativo a 5 e \\
probabilidade, respectivamente. & \\
Médias com letras diferentes, na coluna, diferem estatisticamente $(\mathrm{P}<0,05)$ pelo teste de Tukey, baseado \\
na amplitude total estudentizada.
\end{tabular}

A resposta das variáveis fisiológicas dos bovinos em função do ambiente (sombra e sol) são mostradas na Figura 1. Na sombra, os animais com pelos mais compridos tiveram um aumento na taxa de sudação e uma diminuição da FC, TR e FR. As temperaturas superficiais do corpo aumentaram, com exceção da temperatura da axila. Tem um subgrupo de animais com pelos longos e alta sudação, mas todas as temperaturas baixas. No sol, observouse animais com alta taxa de sudação e baixa temperatura superficial da axila. Observou-se também um subgrupo de animais com pelos mais compridos, maiores taxas de sudação, frequência respiratória, temperatura superficial da garupa e costela. Esses animais aparentaram mecanismos de dissipação de calor que seriam interessantes para seleção, resultados similares ao obtidos por McManus et al. (2011) que mostrou que animais com pelagem mais claras eram melhor adaptados ao ambiente tropical.

As análises canônicas (Figura 2) mostraram uma clara separação do grupo Nelore branco dos outros dois grupos, tanto na sombra quanto no sol, com maior sobreposição dos outros dois grupos, Pantaneiro e Nelore vermelho, ambos de pelagem vermelha. Segundo Caro (2005), as causas adaptativas da variação da coloração da pelagem nos mamíferos ainda não são bem compreendidas, havendo três hipóteses clássicas sobre a importância da função da pelagem, que são a ocultação, comunicação e processo de regulação fisiológica. 
Rev. Bras. Saúde Prod. Anim., Salvador, v.15, n.4, p.854-865 out./dez., 2014 http://www.rbspa.ufba.br ISSN 15199940
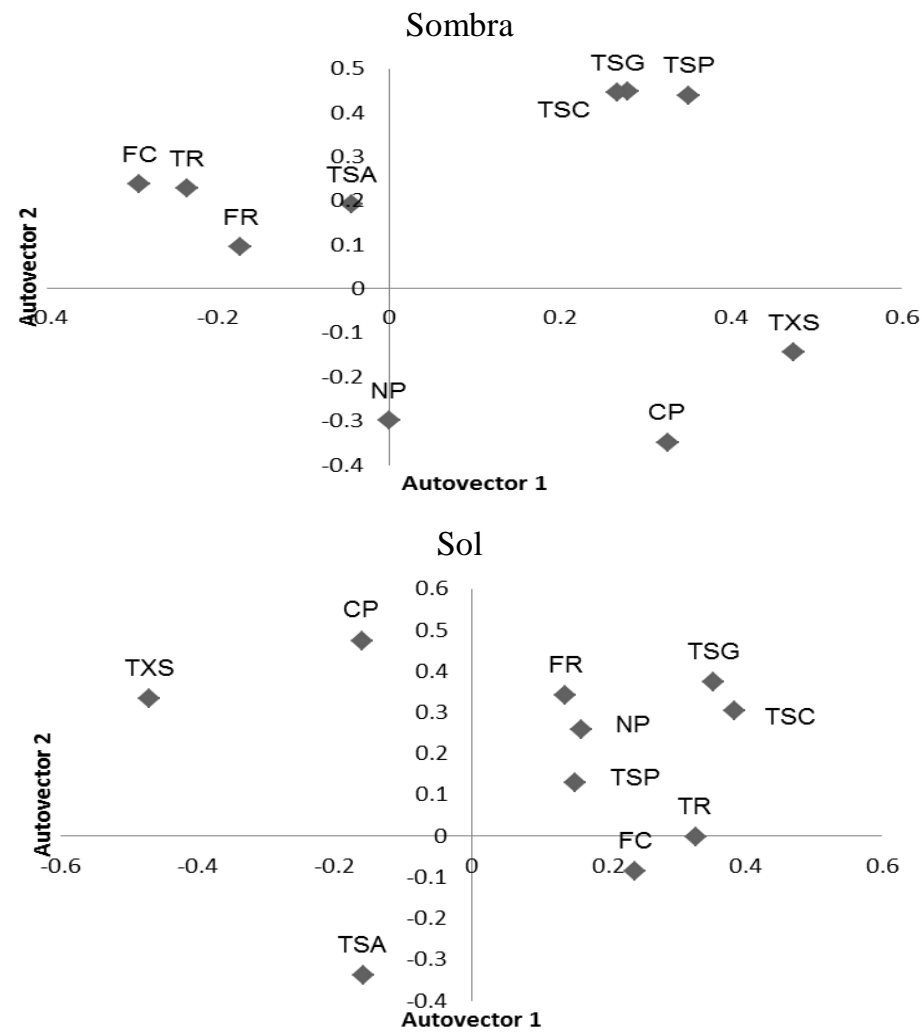

Figura 1. Primeiros dois componentes principais para bovinos no sol e na sombra

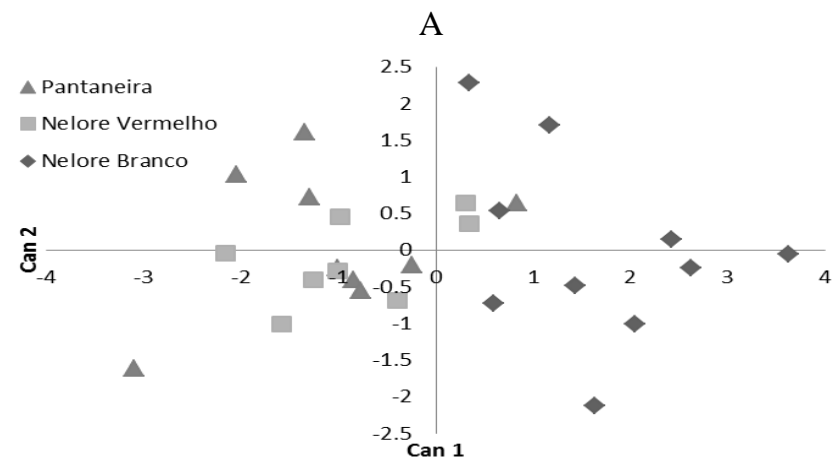

B

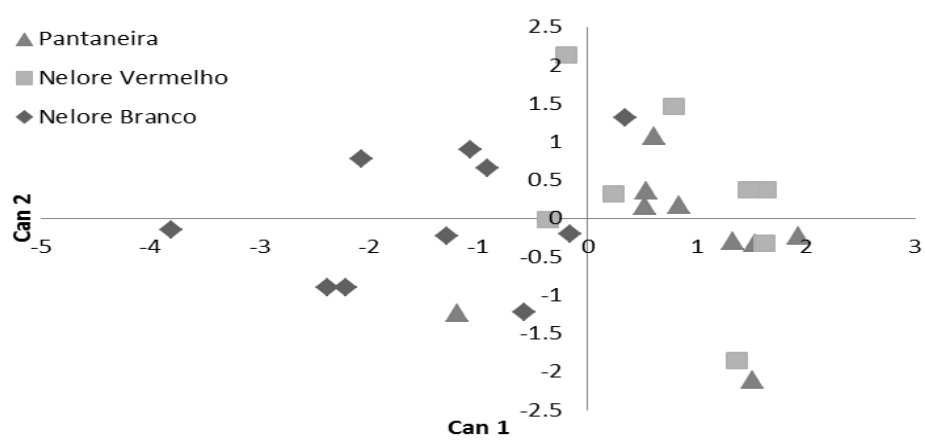

Figura 2. Análises Canônicas por grupos genéticos na Sombra (A) e no Sol (B) 
No sol, as características úteis para separação dos grupos genéticos (Figura 3) incluiram as temperaturas superficiais, TXS e CP, mas não a FR e TR. Na sombra, as temperaturas superficiais não tiveram tanta influência na separação dos grupos.
A partir do método de agrupamento hierárquico foi possível estabelecer dois grupos, tanto na sombra como no sol, um com o Nelore branco e Pantaneiro e outro do Nelore vermelho (Figura 4), o que mostra que ambas as raças (Nelore branco e Pantaneiro) são mais adaptadas ao Pantanal.

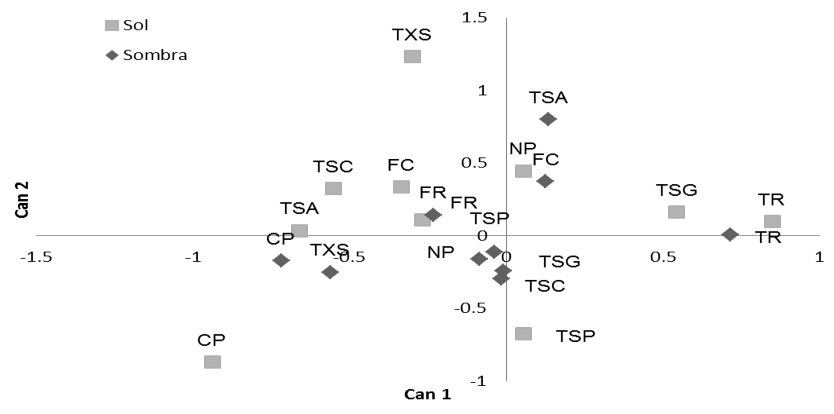

Figura 3. Médias canônicas estandardizadas para características de bovinos no sol e na sombra

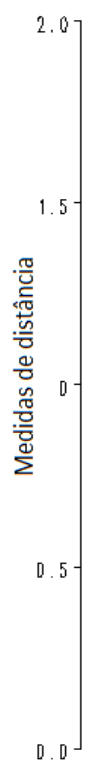

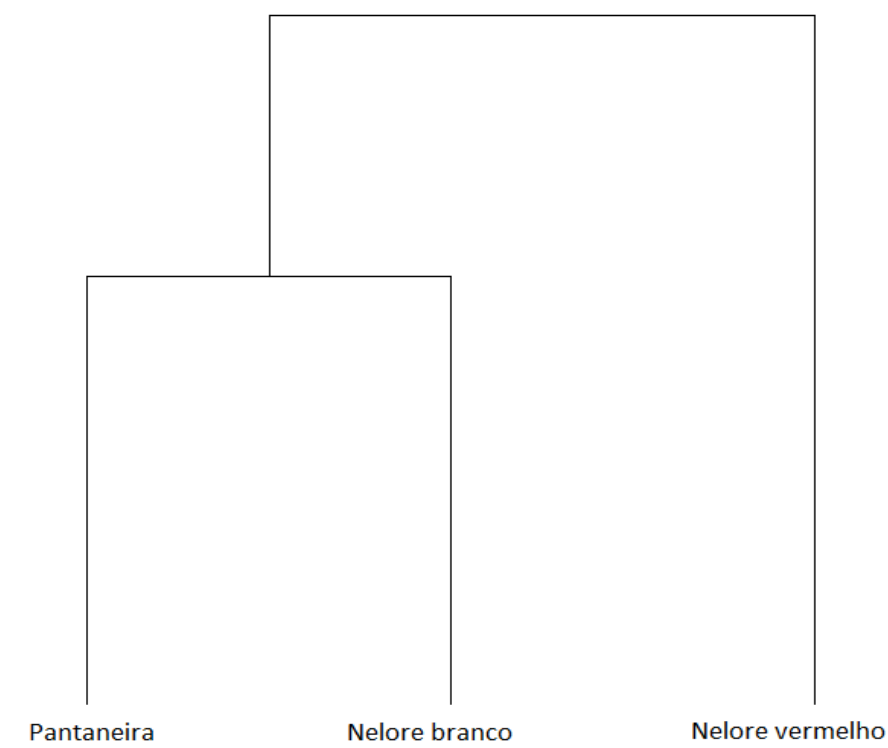

Figura 4. Dendrograma obtido de dados de variáveis fisiológicas e pelagem de três grupos genéticos de bovinos no Pantanal

O Nelore vermelho foi selecionado para tipo de pelagem na fazenda Havaí, Pantanal da Nhecolândia, MS, pelo médico veterinário, Dr. Joaquim Cavalcanti Freire durante 55 anos, em regime fechado, o que provavelmente 
Rev. Bras. Saúde Prod. Anim., Salvador, v.15, n.4, p.854-865 out./dez., 2014 http://www.rbspa.ufba.br ISSN 15199940

aumentou a endogamia. São animais que carregam em sua formação características genéticas e de adaptação ao meio ambiente, desenvolvidas ao longo de vários anos de cruzamentos endogâmicos e seleção natural, devido à pouca interferência do produtor, que apenas tinha o cuidado de mantê-los em invernadas separadas. Esta prática garantiu um número razoável de animais com esta pelagem bem definida, mas com os demais índices zootécnicos foram pouco estudados ou até mesmo desconhecidos (EGITO et al., 2013). Para sua preservação e facilitar os trabalhos de determinação dos índices zootécnicos deste grupo genético, a Embrapa Pantanal adquiriu alguns animais com este tipo de pelagem e atualmente possui um pequeno rebanho na Fazenda Nhumirim para estudos. Em face de todo esse processo de seleção endogâmica, essa linhagem pode ter perdido algumas características de adaptação ao calor.

A avaliação comparativa dos aspectos relacionados à tolerância nos diversos grupos genéticos e raças bovinas, tal como realizado no presente estudo, permite um melhor conhecimento acerca das relações entre características de produção e adaptação, contribuindo, assim, para o aumento da produtividade destas raças por meio de práticas de manejo e técnicas de melhoramento genético, agregando características, tais como rusticidade, resistência às doenças e às condições ambientais menos favoráveis.

Com base nos resultados obtidos no presente estudo, os grupos genéticos Nelore branco e Pantaneiro apresentaram características fisiológicas e tolerância ao calor similar. O Nelore vermelho, por ser uma linhagem que foi selecionada para pelagem, pode ter perdido algumas características de adaptação ao calor, mas também apresentou-se tolerante.
Portanto, os grupos estudados são adaptados às condições de alta temperatura e umidade do Pantanal, garantindo bem-estar aos animais criados extensivamente.

\section{REFERÊNCIAS}

BACCARI JUNIOR, F. Métodos e técnicas de avaliação da adaptabilidade dos animais nos trópicos. In: SEMANA DE ZOOTECNIA, 11., 1986, Pirassunuga. Anais... Pirassununga: Fundação Cargill, 1986. p.53-64.

BIANCHINI, E.; McMANUS, C.; LUCCI, C.M.; FERNANDES, M.C.B.; PRESCOTT, E.; MARIANTE, A.S.; EGITO, A.A. Características corporais associadas com a adaptação ao calor em bovinos naturalizados brasileiros.

Pesquisa Agropecuária Brasileira, v.41, n.9, p.1443-1448, 2006.

CARO, T. The adaptive significance of coloration in mammals. BioScience, v.55, n.2, p.125-136, 2005.

EGITO, A.A.; MARIANTE, A.S.; ALBUQUERQUE, M.S.M. Programa brasileiro de conservação de recursos genéticos animais. Archivos de Zootecnia, v.51, p.39-52, 2002.

EGITO, A.A.; PEDREIRA, M.M.; ROSA, A.N.; SIQUEIRA, F. Caracterização genética de bovinos da raça Nelore Variedade Vermelha por meio de marcadores microssatélites. In: SIMPÓSIO BRASILEIRO DE MELHORAMENTO ANIMAL, 10, 2013, Uberaba. Anais... Uberaba: SBMA, 2013. 
Rev. Bras. Saúde Prod. Anim., Salvador, v.15, n.4, p.854-865 out./dez., 2014 http://www.rbspa.ufba.br ISSN 15199940

FAÇANHA, D.A.E.; CHAVES, D.F.; MORAIS, J.H.G.; VASCONCELOS, A.M.; COSTA, W.P.;

GUILHERMINO, M.M. Tendências metodológicas para avaliação da adaptabilidade ao ambiente tropical.

Revista Brasileira de Saúde e Produção Animal [online], v.14, n.1, p.91-103, 2013.

\section{FEITOSA, F.L.F. Semiologia} veterinária: a arte do diagnóstico. São Paulo: Roca, 2004. 807p.

FITZHUGH, H. A. Animal size and efficiency, with special reference to the breeding female. Animal Production, v.27, p.393-401, 1978.

FURTADO, D.A.; PEIXOTO, A.P.; REGIS, J.E.F.; NASCIMENTO, J.W.B.; ARAUJO, T.G.P.; LISBOA, A.C.C. Termorregulação e desempenho de tourinhos Sindi e Guzerá, no agreste paraibano. Revista Brasileira de Engenharia Agrícola e Ambiental, v.16, n.9, p.1022-1028, 2012.

HANSEN, P.J. Physiological and cellular adaptations of zebu cattle to thermal stress. Animal Reproduction Science, v.82-83, p.349-360, 2004.

JULIANO, R.S.; RAMOS, A.F.; ABREU, U. G.P.; SANTOS, S.A. Análise de características reprodutivas indicadoras de puberdade em tourinhos Pantaneiros. Archivos de Zootecnia, v.60, p.1-4, 2011.

KABUGA, J.D. The influence of thermal conditions on rectal temperature, respiration rate and pulse rate of lactating Holstein-Friesian cows in the humid tropics. International Journal of Biometeorology, v.36, p.146-150, 1992.
KAPS, M.; LAMBERSON, W.R.

Biostatistics for animal science: an introductory text. Wallingford: Cabi Publishing, 2009. 520p.

MAGALHÃES, J.A.; TAKIGAWA, R.M.; TOWNSEND, C.R.; COSTA, N. DE L.; PEREIRA, R.G. DE A. Tolerância de bovídeos à temperatura e umidade do trópico úmido. Revista Científico de Produção Animal, v.2, p.62-167, 2000.

MAZZA, M.C.; TROVO, J.B.F.; SERENO, J.R.B.; SILVA, R.A.M.S.; ABREU, U.G.P. Desempenho de bovinos pantaneiros no núcleo de conservação da fazenda Nhumirim, Nhecolândia, Pantanal: avaliação preliminar. Centro de Pesquisas Agropecuárias do Pantanal. v.11, p.1-5, 1989.

McDOWELL, R.E.; LEE, D.H.K.; FOHRMAN, M.H. The measurement of water evaporation from limited areas of a normal body surface. Journal of Animal Science, v.13, p.405-416, 1954.

McMANUS, C.; PRESCOTT, E.; PALUDO, G. R.; BIANCHINI, E.; LOUVANDINI, H.; MARIANTE, A. S. Heat tolerance in naturalized Brazilian cattle breeds. Livestock Science, v.120, p.256-264, 2009.

McMANUS, C.; CASTANHEIRA, M.; PAIVA, S.R.; LOUVANDINI, H.; FIORAVANTI, M.C.; PALUDO, G.R.; BIANCHINI, E.; CORRÊA, P.S. Use of multivariate analyses for determining heat tolerance in Brazilian cattle. Tropical Animal Health and Production, v.33, n.3, p.623-630, 2011.

ROBINSON, E.N. Termorregulação. In: CUNNINGHAM, J.G. Tratado de fisiologia veterinária. 2 . ed. Rio de Janeiro: Guanabara Koogan, 1999. p.427435. 
Rev. Bras. Saúde Prod. Anim., Salvador, v.15, n.4, p.854-865 out./dez., 2014 http://www.rbspa.ufba.br ISSN 15199940

SANTOS, S. A.; McMANUS, C.; SOUZA, G. S.; SORIANO, B.M.A.; SILVA, R.A.M.S.; COMASTRI FILHO, J.A.; ABREU, U.G.P.; GARCIA, J.B. Variações da temperatura corporal e da pele de vacas e bezerros das raças Pantaneira e Nelore no Pantanal. Archivos de Zootecnia, v.54, p.237-244, 2005.

\section{SAS Institute. System for Microsoft Windows. Cary, 2010.}

SCHLEGER, A.V.; TURNER, H. G. Sweating rates of cattle in the field and their reaction to diurnal and seasonal changes. Australian Journal of Agricultural Research, v.16, p. 92106. 1965.

\section{SILVA, R.G. Introdução à}

Bioclimatologia Animal. São Paulo: Nobel, 2000. 286p.

SORIANO, B.M.; GALDINO, S. Análise da distribuição da frequiência mensal de precipitação para a Subregião da Nhecolândia, Pantanal, Mato Grosso do Sul, Brasil. Boletim de Pesquisa e Desenvolvimento, v.34, p.1-23, 2002.

SOUZA, B.B.; ALFARO, C.E.P. O Sindi e sua adaptação ao semiárido paraibano. Agropecuária Tropical, n.150, p.64-66, 2006.

TITTO, C.G.; TITTO, E.A.L.; VIEIRA, R.V.; GLASER, F.D.; TITTO, R.M.; ABLAS, D.S.; PEREIRA, A.M.F.; CUNHA LEME, T.M.; GATTO, E.G.; RAINERI, C. Tolerância ao calor em bovinos de corte de raças europeias utilizadas em cruzamentos industriais no Brasil. In: CONGRESSO BRASILEIRO DE BIOMETEOROLOGIA, 4., 2006, Ribeirão Preto, São Paulo. Anais... Ribeirão Preto: SBBiomet, 2006.
VERCOE, J. E.; FRISCH, J.E. Genotype (breed) and environment interaction with particular reference to cattle in the tropics. Australian Journal of Applied Sciences, v.5, p.401-409, 1992.

VIA, S.; LANDE, R. Genotypeenvironment interactions and the evolution of phenotypic plasticity. Evolution, v.39, n.3, p.505-522, 1985.

VIEIRA, R.V. Teste de tolerância ao calor em bovinos de corte de raças européias utilizadas no cruzamento industrial no Brasil. 2003. 43f. Dissertação (Mestrado) - Faculdade de Zootecnia e Engenharia de Alimentos, Universidade de São Paulo, Pirassununga.

WENZ, J.R., MOORE, D.A.; KASIMANICKAM, R. Factors associated with the rectal temperature of Holstein dairy cows during the first 10 days in milk. Journal of Dairy Science, v.94, p.1864-1872, 2011.

WILSON, D.C.S.; CORBETT, A.D.; BOVELL, D.L. A preliminary study of the short circuit current (Isc) responses of sweat gland cells from normal andanhidrotic horses to purinergic and adrenergic agonists. Journal Compilation, v.18, p.152-160, 2007.

Data de recebimento: 18/02/2014

Data de aprovação: 31/10/2014 